\title{
Os empregados da agricultura brasileira: diferenciais e determinantes salariais
}

Marina Silva da Cunha ${ }^{1}$

Resumo: Este trabalho estuda os diferenciais e os determinantes salariais dos empregados na agricultura brasileira, no período de 1981 a 2005, com base nas informações das Pesquisas Nacionais por Amostra de Domicílios (PNAD), do IBGE. Foi utilizada a metodologia de Heckman para corrigir viés de seleção, comum em equações salariais estimadas por Mínimos Quadrados Ordinários, além da decomposição de Oaxaca-Blinder, para analisar os diferenciais salariais entre 1981 e 2005. Entre os determinantes salariais, foram analisados: educação, experiência, gênero, região, tempo semanal de trabalho e carteira de trabalho, além de alguns determinantes macroeconômicos. Os resultados do trabalho sugerem uma redução dos diferenciais salariais, principalmente no final do período estudado, e que as variáveis educação, idade e carteira de trabalho contribuíram para reduzir os diferencias salariais entre 1981 e 2005; e por outro lado, as variáveis gênero, região geográfica e tempo semanal de trabalho, contribuíram para aumentá-los. Além disso, as variáveis macroeconômicas incluídas na análise foram estatisticamente significativas na explicação dos diferenciais salariais agrícolas.

${ }^{1}$ Doutora em Economia Aplicada pela Esalq/USP e professora do Departamento de Economia da UEM. 
Palavras-chave: salários, Heckman, decomposição de Oaxaca.

Classificação JEL: J31, J43

Abstract: This work studies the wage differentials and determinants of the employees in Brazilian agriculture, in period 1981-2005, on the basis of the information of the National Research for Sample of Domiciles of the IBGE. The methodology of Heckman is used to correct bias of selection, common in wage equations gotten by Least Ordinary Square, besides the decomposition of Oaxaca-Blinder, to analyze the wage differentials between 1981 and 2005. Between wage determinants, had been analyzed: education, experience, region, weekly working time and formal work, besides some macroeconomic determinants. The results of the work suggest a reduction of the wage differentials, mainly in the end of the studied period, and that the variable education, age and formal work had contributed to reduce the wage differentials between 1981 and 2005; and on the other hand, the variable geographic region and weekly working time, had contributed to increase them. Moreover, the macroeconomic variables included in the analysis were statistical significant in the explanation of the agricultural differentials.

Key words: wages, Heckman, decomposition of Oaxaca.

JEL Classification: J31, J43

\section{Introdução}

O aumento da desigualdade salarial, tanto nos países desenvolvidos quanto nos em desenvolvimento, tem despertado a atenção de pesquisadores para o estudo do tema, principalmente entre trabalhadores qualificados e menos qualificados ${ }^{2}$. As explicações para esse fato têm sido atribuídas, especialmente, ao processo de liberalização econômica

\footnotetext{
${ }^{2}$ Ver Feenstra \& Hanson (1996) e Sachs \& Shatz (1996), para os Estados Unidos; Hanson \& Harrison (1999) e Cragg \& Epelbaum (1996), para o México; Beyer et al. (1999), Romaguera (1991) e Gindling \& Robbins (2001), para o Chile; Attanasio et al. (2003), para a Colômbia; entre outros.
} 
(Wood, 1997) e às mudanças tecnológicas (Machin \& Reenen, 1998). No entanto, como adverte Wood (1997), como a abertura econômica também propicia mudanças no padrão tecnológico, via maior acesso a bens de capital, idéias e tecnologias, uma combinação de ambas as explicações parece ser a melhor justificativa para tais modificações no mercado de trabalho.

Existem vários trabalhos sobre os diferenciais e determinantes salariais para a economia brasileira. Conforme Green et al. (2001) e Arbache et al. (2004), ocorreu um aumento da demanda e dos prêmios salariais para os trabalhadores mais qualificados associados à abertura comercial brasileira. A desigualdade salarial, contudo, se manteve praticamente estável - houve apenas uma pequena redução ${ }^{3}$.

Na escassa literatura recente sobre os salários agrícolas, podem ser citados os trabalhos de Staduto et al. (2002), Istake \& Bacha (1999) e Bacha (1991), os quais têm como foco os determinantes salariais ${ }^{4}$. Entre as variáveis explicativas utilizadas, destacam-se a produtividade do trabalho e o salário mínimo. O presente estudo procura contribuir para a discussão do tema, incorporando variáveis relevantes na determinação dos salários agrícolas, relacionadas às características pessoais produtivas e não-produtivas dos indivíduos, o que é possível por meio da utilização das informações das PNADs, não utilizadas nos trabalhos citados para o setor.

Este trabalho também incorpora outros determinantes conjunturais, tais como o desemprego e o câmbio, buscando captar os reflexos das importantes transformações da economia brasileira nas últimas décadas sobre os salários dos empregados do setor agrícola. Desse modo, esse estudo tem como objetivo analisar os diferenciais e os determinantes salariais dos empregados da agricultura brasileira, no período de 1981 a 2005. Ressalta-se que esse setor tem sofrido o impacto dessas mudanças

\footnotetext{
${ }^{3}$ Outros trabalhos também vêm abordando esse tema, como por exemplo: Menezes-Filho et al. (2001); Souza (2004); Machado \& Jayme (2003) e Coelho \& Corseuil (2002). ${ }^{4}$ Esses trabalhos utilizaram modelos de equilíbrio do mercado de trabalho para estimar equações de determinação dos salários, sendo que Bacha (1991) e Istake (1999) realizaram análises regionais; o primeiro sobre os salários agrícolas para a cafeicultura de Minas Gerais, no período 1969-1985, e o segundo para o Paraná, entre 1977 e 1996. Stadudo et al. (2002) realizou uma análise para a agricultura brasileira no período de 1971 a 1996.
} 
e tem desempenhado um importante papel na redução da inflação (via crescimento da oferta de produtos) e na geração de divisas (por meio do crescimento das exportações), o que não seria possível sem uma melhoria da eficiência e da produtividade. Além disso, conforme destaca Helfand \& Rezende (2001), o impacto das reformas políticas adotadas no setor agrícola foi altamente diferenciado entre produtos (exportáveis e importáveis), regiões geográficas, estabelecimentos e ao longo do tempo.

O presente estudo está organizado em cinco tópicos. Além desta introdução, na seção 2, foram expostas as informações utilizadas no trabalho, buscando caracterizar os empregados na agricultura brasileira. Na seção 3, foi apresentada a metodologia para a análise dos diferenciais salariais e, posteriormente, na seção 4, os resultados deste trabalho. Por fim, na seção 5, tem-se a conclusão do trabalho.

\section{Os empregados na agricultura brasileira}

Os empregados do setor agrícola brasileiro foram analisados neste trabalho tomando como base as informações anuais das Pesquisas Nacionais por Amostra de Domicílios (PNADs), no período de 1981 a 2005 - apenas a partir de 1981 que foi permitido uma análise mais aprofundada e sistemática do perfil distributivo dos salários, como a escolaridade dos indivíduos ${ }^{5}$. Foram utilizadas as informações dos indivíduos com rendimento positivo, com 15 anos ou mais de idade e trabalhando 15 horas ou mais por semana. Ademais, para viabilizar as estimações, foram considerados os indivíduos com informações disponíveis de idade, escolaridade, tempo de trabalho semanal e carteira de trabalho ${ }^{6}$.

Para permitir uma comparação dos resultados ao longo de todo o período, para os anos de 1992 a 2005, incluiu-se, na região CentroOeste, o estado do Tocantins, que foi desmembrado do antigo estado de Goiás e é atualmente classificado, geograficamente, na região Norte. Além disso, buscando também uniformizar as informações, foi excluída da análise a área rural da região Norte em 2005, uma vez que, nos anos anteriores, esta área não foi pesquisada nas PNADs.

\footnotetext{
${ }^{5}$ Balsadi (2007) utilizou as informações das PNADs para estudar a qualidade do emprego no setor agrícola no período 2001-2004.

${ }^{6}$ Excepcionalmente, nos anos de 1991, 1994 e 2000 a pesquisa não foi realizada.
} 
Todas as estimativas foram realizadas utilizando os fatores de expansão presentes nas PNADs, sendo que, para os anos de 1992 a 1996, foram utilizados os fatores corrigidos e disponibilizados no ano de 1997, para o ano de 1999, os novos fatores apresentados em 2001 e, para os anos de 2001 a 2003, os respectivos fatores contidos em 2004. Foi considerado como salário os rendimentos obtidos em todos os trabalhos, deflacionados pelo Índice Nacional de Preços ao Consumidor (INPC), do IBGE, com base no mês de setembro de 2005.

Conforme as informações das PNADs, a composição setorial do emprego tem se modificado nas últimas décadas, com a redução dos postos de trabalho da agricultura, processo típico em economias em desenvolvimento, conforme pode se observar na Tabela 1. O setor agrícola representava $16,6 \%$ dos postos de trabalho em 1981 e 10,2\% em 2005. A população estimada total dos empregados, com informações disponíveis, era de 26.558.485 em 1981 e de 47.055.009 em 2005.

A Tabela 2 traz algumas características dos indivíduos empregados na agricultura e na economia brasileira, para o período 1981-2005. A escolaridade média no setor agrícola é tradicionalmente mais baixa que a média brasileira. Em 2005, esse indicador era igual a 3,54 anos de estudos no setor agrícola, enquanto que no Brasil, era de 8,16 anos. No entanto, a escolaridade vem aumentando significativamente na agricultura, praticamente dobrando no período em análise, com alta de 114,5\%. Conforme a Figura 1, houve uma redução das pessoas analfabetas ou com menos de um ano de escolaridade (Nível 1), como também daquelas com primário incompleto (Nível 2), em detrimento dos demais níveis na agricultura.

A idade média de empregados na agricultura é maior que a da média nacional e teve poucas alterações, com um aumento de pouco mais de um ano entre 1981 e 2005, alcançando 34,7 anos no último em 2005.

Contrariando uma tendência da economia brasileira, na qual a mulher vem ganhando espaço no mercado de trabalho, na agricultura, a participação da mulher reduziu no período estudado, com apenas $10,25 \%$ em 2005. Por outro lado, a participação da mulher no mercado de trabalho brasileiro foi de $43,36 \%$ em $2005^{7}$.

\footnotetext{
${ }^{7}$ Vale notar que, no setor de serviços, a participação das mulheres é superior à dos homens, e esse fato ocorreu pela primeira vez nesse setor em 1988, conforme informações das PNADs.
} 
Tabela 1 - Evolução da população e da amostra, após a aplicação cumulativa das restrições, 1981-2005

\begin{tabular}{|c|c|c|c|c|}
\hline \multirow{3}{*}{ Ano } & \multicolumn{4}{|c|}{ População Economicamente Ativa Ocupada } \\
\hline & \multicolumn{2}{|c|}{ Brasil } & \multicolumn{2}{|c|}{ Agricultura } \\
\hline & População & Amostra & População & Amostra \\
\hline 1981 & 26.558 .485 & 112.874 & 4.403 .613 & 14.172 \\
\hline 1982 & 27.509 .660 & 116.326 & 4.679 .267 & 15.766 \\
\hline 1983 & 28.710 .740 & 117.913 & 4.570 .004 & 15.231 \\
\hline 1984 & 29.205 .307 & 117.751 & 5.349 .048 & 17.917 \\
\hline 1985 & 31.506 .124 & 124.879 & 5.391 .801 & 17.960 \\
\hline 1986 & 33.331 .463 & 71.107 & 5.128 .297 & 9.635 \\
\hline 1987 & 34.523 .135 & 74.345 & 5.126 .549 & 9.715 \\
\hline 1988 & 35.278 .515 & 74.120 & 5.056 .010 & 9.452 \\
\hline 1989 & 36.412 .567 & 75.940 & 4.810 .120 & 8.856 \\
\hline 1990 & 35.195 .756 & 76.582 & 4.780 .593 & 9.018 \\
\hline 1992 & 35.223 .467 & 77.594 & 4.706 .069 & 9.332 \\
\hline 1993 & 36.074 .126 & 79.165 & 4.572 .360 & 8.946 \\
\hline 1995 & 37.626 .702 & 83.518 & 4.481 .412 & 8.905 \\
\hline 1996 & 37.934 .286 & 82.197 & 4.253 .421 & 8.189 \\
\hline 1997 & 32.865 .025 & 73.477 & 3.423 .565 & 6.854 \\
\hline 1998 & 38.742 .244 & 85.378 & 3.992 .264 & 7.787 \\
\hline 1999 & 40.004 .858 & 86.744 & 4.280 .411 & 8.303 \\
\hline 2001 & 43.768 .009 & 96.943 & 4.078 .821 & 8.344 \\
\hline 2002 & 42.547 .127 & 93.930 & 4.293 .326 & 8.756 \\
\hline 2003 & 43.164 .024 & 93.593 & 4.402 .158 & 8.933 \\
\hline 2004 & 45.802 .700 & 98.845 & 4.559 .214 & 9.152 \\
\hline 2005 & 47.055 .009 & 102.465 & 4.586 .668 & 9.325 \\
\hline
\end{tabular}

Fonte: Elaborado com base nas PNADs. ${ }^{a}$ A estimativa da população é obtida utilizando o fator de expansão disponibilizado pelo IBGE.

O deslocamento espacial das pessoas empregadas na agricultura brasileira aponta para uma redução relativa nas regiões Sudeste e Sul em relação às demais, em especial para a região Centro-Oeste, que aumentou sua participação em quase 4 pontos percentuais, o que reflete a expansão do setor nesta região. Pode-se dizer, contudo, que o emprego no setor agrícola se expande nas regiões Nordeste, Norte e Centro-Oeste, onde se encontram áreas tanto de fronteira como de alto grau de tecnificação, como a soja no Centro-Oeste e a fruticultura no Nordeste. 
Tabela 2 - Características das pessoas empregadas na economia brasileira, por setor de ocupação, entre 1981 e 2005

\begin{tabular}{rrrrrrrrr}
\hline & \multicolumn{4}{c}{ Brasil } & \multicolumn{7}{c}{ Agricultura } \\
\cline { 2 - 9 } & 1981 & 1985 & 1995 & 2005 & 1981 & 1985 & 1995 & 2005 \\
\hline Escolaridade & & & & & & & & \\
Nível 1 & 17,32 & 15,49 & 11,21 & 6,43 & 47,89 & 46,68 & 39,47 & 26,66 \\
Nível 2 & 20,41 & 18,21 & 14,58 & 8,70 & 31,75 & 30,14 & 29,00 & 24,83 \\
Nível 3 & 33,61 & 33,92 & 33,65 & 24,33 & 18,29 & 20,60 & 27,13 & 34,40 \\
Nível 4 & 12,08 & 13,18 & 15,70 & 18,21 & 1,44 & 1,72 & 2,97 & 9,24 \\
Nível 5 & 12,07 & 14,03 & 18,20 & 33,29 & 0,51 & 0,72 & 1,14 & 4,59 \\
Nível 6 & 4,50 & 5,17 & 6,65 & 9,04 & 0,13 & 0,14 & 0,29 & 0,28 \\
Média & 5,16 & 5,56 & 6,44 & 8,16 & 1,65 & 1,80 & 2,30 & 3,54
\end{tabular}

Idade

Média $\quad 31,51 \quad 31,53 \quad 32,90 \quad 34,23 \quad 33,24 \quad 32,80 \quad 33,76 \quad 34,70$ Sexo

$\begin{array}{ccccccccc}\text { Homem } & 68,56 & 66,02 & 60,32 & 56,64 & 86,96 & 85,95 & 89,44 & 89,75 \\ \text { Mulher } & 31,44 & 33,98 & 39,68 & 43,36 & 13,04 & 14,05 & 10,56 & 10,25\end{array}$

Região

$\begin{array}{lllllllll}\text { Nordeste } & 22,70 & 22,25 & 21,95 & 22,38 & 37,01 & 40,69 & 36,30 & 41,17\end{array}$

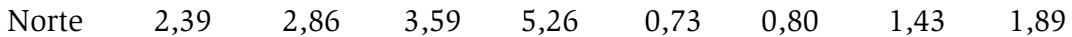

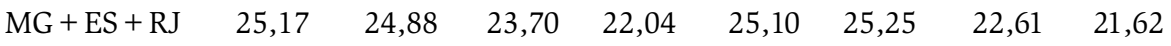

$\begin{array}{lllllllll}\text { São Paulo } & 27,97 & 28,36 & 26,70 & 25,55 & 14,60 & 13,27 & 14,69 & 13,57\end{array}$

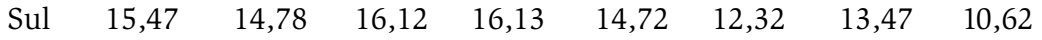

$\begin{array}{lllllllll}\text { Centro-Oeste } & 6,30 & 6,88 & 7,94 & 8,63 & 7,84 & 7,67 & 11,51 & 11,13\end{array}$

Tempo

semanal de

trabalho

$\begin{array}{rrrrrrrrr}\text { '15h a 39h' } & 11,77 & 11,15 & 14,71 & 17,36 & 8,58 & 5,54 & 6,74 & 11,78 \\ \text { '40h a 44h' } & 27,39 & 26,48 & 42,24 & 43,22 & 20,06 & 20,14 & 31,40 & 35,44 \\ \text { '45h a 48h' } & 32,79 & 34,87 & 21,70 & 20,24 & 36,09 & 33,28 & 24,99 & 24,01 \\ \text { ' } \geq 49 \text { ' } & 28,06 & 27,50 & 21,35 & 19,18 & 35,26 & 41,03 & 36,87 & 28,77\end{array}$

Carteira de

trabalho

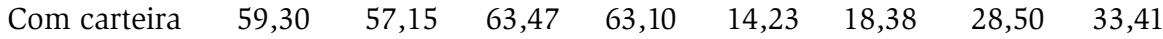

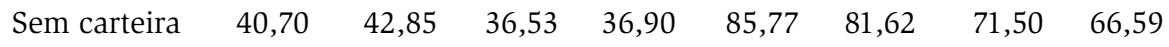

Fonte: Elaborado com base nas PNADs. 
Figura 1 - Composição das pessoas ocupadas no setor agrícola, segundo o nível educacional, 1981-2005

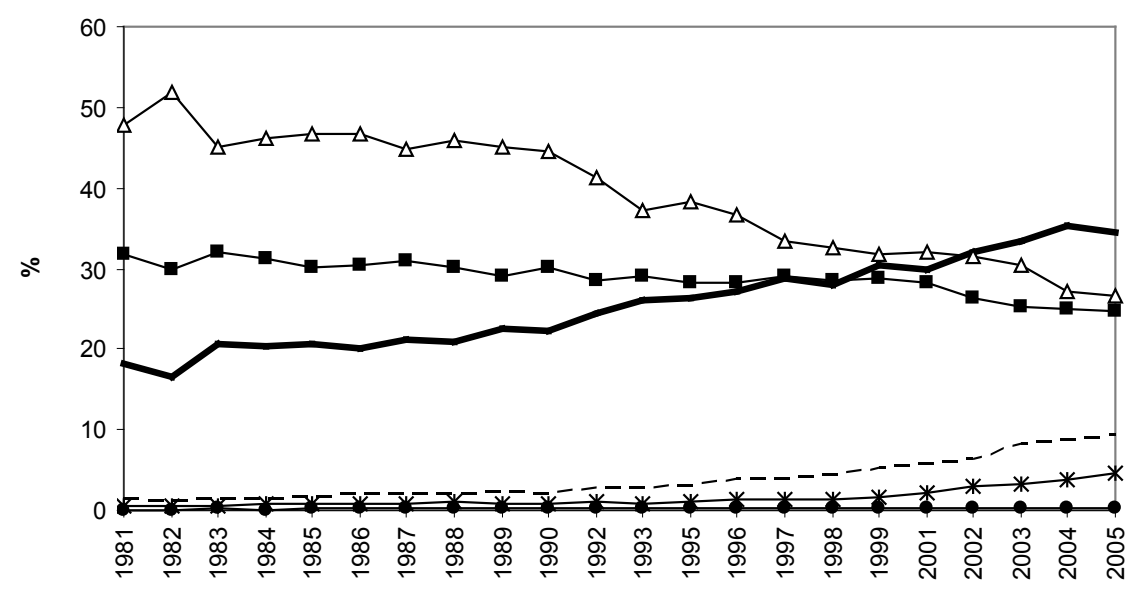

$\Delta-$ Nível $1 \longrightarrow$ Nível 2

Nível 3 - - - Nível $4 \longrightarrow$ - Nível 5

Nível 6

Fonte: Elaborado com base nas PNADs.

Considerando o tempo semanal de trabalho, nota-se um aumento na proporção dos empregados com tempo de trabalho de 15 a 44 horas semanais, e uma redução nos segmentos com mais horas semanais de trabalho.

No Brasil, observa-se uma expansão da proporção das pessoas ocupadas com carteira de trabalho no período, atingindo 66,6\% em 2005. O setor agrícola também seguiu essa tendência, apesar de ser o ramo de atividade com o menor grau de formalização na economia brasileira.

O setor agrícola manteve, ao longo desse período, um salário real médio (set $/ 2005=100$ ) inferior ao da média nacional, como pode ser observado na Tabela 3. No ano de 2005, a média dos salários reais da agricultura foi de $\mathrm{R} \$ 347,74$, enquanto que a da economia, de $\mathrm{R} \$ 667,51$. É possível observar os reflexos do Plano Cruzado, no ano de 1986, e do Plano Real, na segunda metade dos anos 1990, nos salários. Embora tenha ocorrido uma redução no salário médio do setor agrícola, a partir de 1999, esta não foi tão significativa quanto à observada na economia, o que pode ser explicado pelo efeito positivo da desvalorização cambial 
ocorrida em 1999 no setor. Nota-se que, em 2004 e 2005, o salário real médio desse setor já superava o observado em 1999.

A Tabela 3 também mostra os índices de desigualdade de Gini, T de Theil e L de Theil, permitindo verificar como os salários estão distribuídos entre os empregados no período de 1981 a $2004^{8}$. O maior valor para o índice de Gini na economia brasileira ocorreu em 1989 e o menor, em 2005, sinalizando uma tendência negativa nessa medida de desigualdade. Por outro lado, a agricultura apresentou o maior valor para esse índice em 1988 e o menor, em 1983. Todavia deve-se ressaltar que o segundo menor valor obtido nesta série foi em 2004. Na agricultura, a média para o índice de Gini nas duas primeiras décadas foi de 0,362 e de 0,368, respectivamente, e, na metade desta nova década, de 0,350, o que também sugere uma redução da desigualdade salarial no período recente. Em 2005, o valor do índice de Gini para a economia brasileira e para a agricultura foi, respectivamente, de 0,486 e de 0,353. Apesar de uma maior oscilação, os índices de desigualdade de Theil indicam os mesmos resultados do índice de Gini.

Vale notar que, idealmente, espera-se que o crescimento do salário real médio seja acompanhado por sua melhor distribuição, o que nem sempre é observado no País. Por exemplo, entre 2004 e 2005, os salários médios cresceram tanto no Brasil quanto na agricultura. No entanto, as medidas de desigualdade diminuíram no Brasil, mas cresceram na agricultura, ou seja, ocorreu um crescimento desigual no setor agrícola entre 2004 e 2005.

\section{Metodologia}

Para obter os diferenciais dos salários, são estimadas equações salariais, utilizando o método dos mínimos quadrados. Contudo, é comum nesses casos a existência de viés de seleção amostral, pelo fato de apenas indivíduos ocupados serem analisados, o que pode levar a uma amostra não aleatória, visto que os indivíduos desempregados ou inativos podem ter características distintas daqueles ocupados. Para contornar esse problema, utiliza-se o procedimento de Heckman (1979). Conforme Greene (2002), inicialmente estima-se a equação de seleção, com a probabilidade de o indivíduo estar trabalhando, por meio do modelo probit, que permite estimar $\lambda$ na equação:

\footnotetext{
${ }^{8}$ Esses índices foram obtidos seguindo Hoffmann (1998).
} 


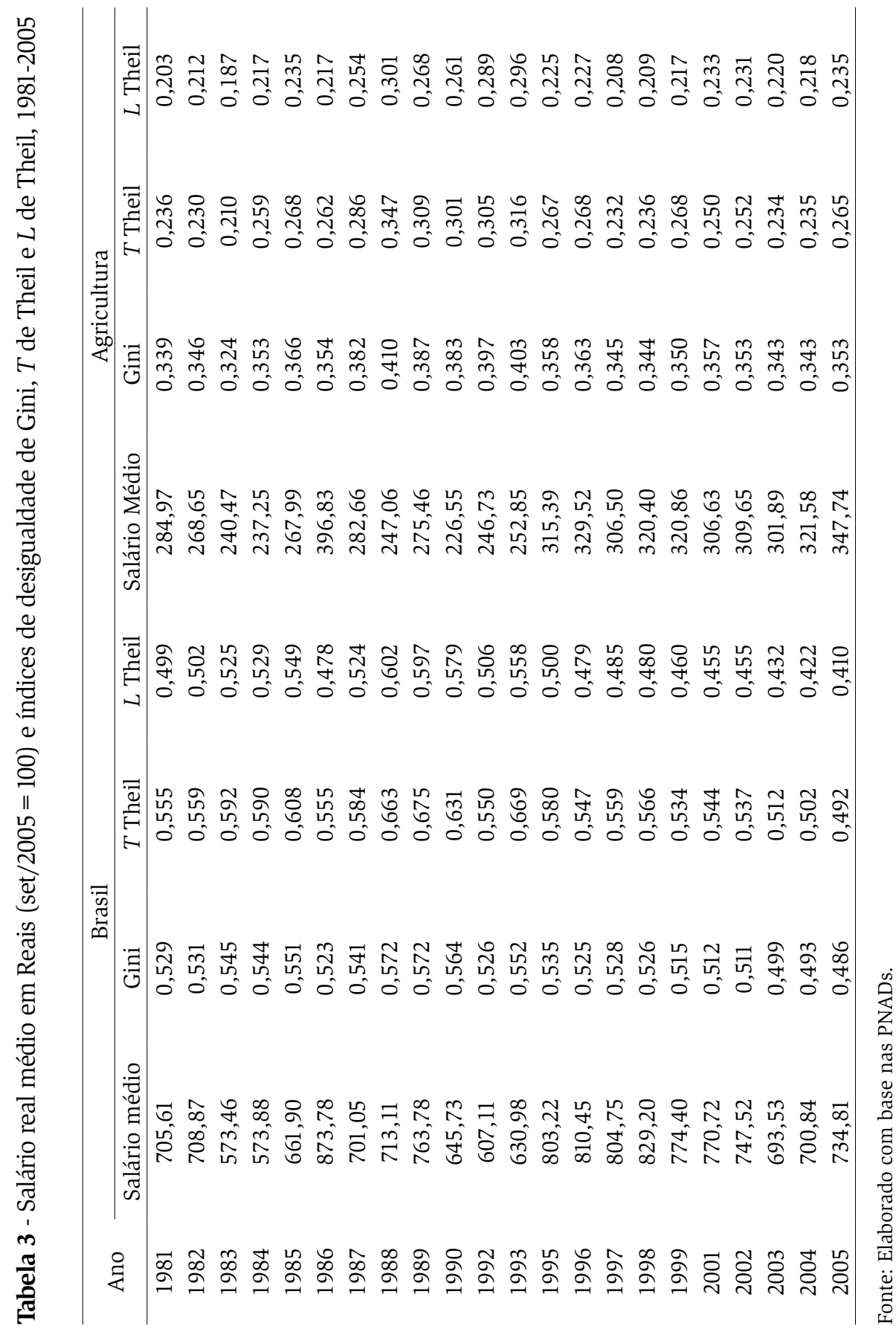




$$
z_{i}^{*}=\mathbf{y}_{i}^{\prime} \boldsymbol{\lambda}+u_{i}
$$

em que, $z_{i}=1$ se $z_{i}^{*}>0$ e $z_{i}=0$ se $z_{i}^{*} \leq 0$. Sendo os salários $\left(w_{i}\right)$ observados apenas se $z=1$.

$$
w_{i}=\mathbf{x}_{i} \beta^{\prime}+\varepsilon_{\mathrm{i}}
$$

com $\left(u_{i}, \varepsilon_{i}\right) \sim$ normal bivariada $\left[0,0,1, \sigma_{\varepsilon}, \rho\right]$. Em seguida, para cada amostra selecionada, calcula-se a razão de Mill invertida:

$$
\hat{\lambda}_{i}=\frac{\phi\left(\mathbf{y}_{i}, \hat{\lambda}\right)}{\Phi\left(\mathbf{y}_{i}^{,} \hat{\lambda}\right)}
$$

Heckman (1979) mostra que, adicionando $\hat{\lambda}_{i}$ como regressor adicional na equação de salários e estimando-a por mínimos quadrados ordinários, obtém-se um estimador consistente dos parâmetros, contornando o problema do viés de seleção, ou seja,

$$
E\left[w_{i} \mid z_{i}=1\right]=\mathbf{x}_{i}^{\prime} \boldsymbol{\beta}+\beta_{\lambda} \lambda\left(\mathbf{y}_{i}^{\prime} \boldsymbol{\lambda}\right)
$$

Em que, $\beta_{\lambda}=\rho \sigma_{\varepsilon}$. Nesse modelo, a variável dependente $\left(w_{i}\right)$ é o logaritmo neperiano dos salários das pessoas empregadas no setor agrícola. O ajustamento das equações é feito por mínimos quadrados ponderados, utilizando como fator de ponderação o peso ou o fator de expansão associado a cada pessoa da amostra.

No vetor de variáveis explicativas $\left(\mathbf{y}_{i}\right)$, estão incluídos educação, experiência, sexo e região geográfica; o vetor $\left(\mathbf{x}_{i}\right)$ inclui, além destes, tempo semanal de trabalho e carteira de trabalho, observados apenas para os indivíduos ocupados. A educação é reconhecida como um fator positivo e relevante na determinação dos rendimentos. Para captar o nível educacional, foi utilizada como proxy o número de anos de escolaridade formal de cada pessoa. Dessa forma, foram incluídas cinco variáveis binárias para distinguir seis níveis educacionais: Nível 1: analfabeto ou com menos de um ano de estudo (tomado como base); Nível 2: alguma educação elementar (de um a três anos de estudo); Nível 3: educação elementar completa ou primário incompleto (quatro a sete anos de estudo); Nível 4: primário completo ou secundário 
incompleto (oito a 10 anos de estudo); Nível 5: secundário completo ou superior incompleto (11 a 14 anos de estudo); Nível 6: superior completo ou mais (15 anos ou mais de estudo). A idade é considerada como uma boa proxy para captar a contribuição do treinamento e da experiência das pessoas. Assim, foi incluída a idade da pessoa, medida em dezenas de anos, e também o quadrado dessa variável, tendo em vista que a renda não varia linearmente com a idade e, sim, por meio de uma relação côncava. Se os coeficientes associados à idade e à idade ao quadrado forem, respectivamente, $b_{1}$ e $b_{2}$, então a idade para o qual o salário esperado é máximo seria obtida quando - $b_{1} /$ $\left(2 b_{2}\right)$, com $b_{1}>0$ e $b_{2}<0$. Para captar a diferença de salário entre os sexos masculino e feminino incluiu-se uma variável binária para sexo, que assume valor 1 para homens. Foram introduzidas cinco variáveis binárias (para distinguir as seis regiões geográficas: Nordeste - tomada como base; Norte; Minas Gerais, Espírito Santo e Rio de Janeiro; São Paulo; Sul e Centro-Oeste) para representar as diferenças regionais do País. Para distinguir os diferentes salários decorrentes do tempo semanal de trabalho, foram utilizadas três variáveis binárias para diferenciar quatro faixas de tempo semanal de trabalho: 15 a 39 horas (tomada como base); 40 a 44 horas; 45 a 48 horas e 49 horas ou mais. Por fim, foi incluída uma variável binária para as pessoas com carteira de trabalho, para distinguir dos salários daquelas sem carteira de trabalho.

Em seguida, para avaliar o quanto dos diferenciais salariais, entre os anos de 1981 e 2005, podem ser explicados por diferenças nas variáveis observadas ou mudanças nos coeficientes e o quanto se deve a diferenças temporais nas características dos indivíduos, utiliza-se a decomposição proposta por Oaxaca (1973) e Blinder (1973). Essa decomposição pode ser apresentada em duas etapas. Na primeira, estimam-se as equações salariais, definidas em (4), para os dois anos analisados ( $a=2005$ e $b=1981$ ) e, na segunda, calcula-se a diferença entre as duas equações nos pontos médios das variáveis, obtendo-se a seguinte expressão:

$$
\bar{W}_{a}-\bar{W}_{b}=\left(\alpha_{a}-\alpha_{b}\right)+\sum_{i} \bar{X}_{i b}\left(\beta_{i a}-\beta_{i b}\right)+\sum_{i} \beta_{i a}\left(\bar{X}_{i a}-\bar{X}_{i b}\right)
$$


Em que $\bar{W}_{a}-\bar{W}_{b}$ é a diferença dos salários médios. O primeiro termo do lado direito corresponde à diferença de interceptos, que indica a diferença residual. Com isso, uma diferença positiva sugere que os indivíduos em a eram mais bem pagos do que em $b$, para qualquer valor das variáveis explicativas. O segundo termo é a parte da diferença de salários que se deve às mudanças nos atributos ou nos coeficientes; assim, se for positiva, o atributo passou a ser mais valorizado. Esses dois primeiros termos correspondem à parcela não explicada pela diferença de atributos. O terceiro termo corresponde ao diferencial explicado e se deve às diferenças de características ponderadas pelo valor que se dá a estas no período inicial, ou seja, que independe da mudança temporal, também denominado de efeito composição.

Por fim, uma crítica que poderia ser feita ao modelo anterior é que não foram agrupados todos os anos, a fim de captar o efeito de variáveis conjunturais nos salários. Com esse intuito, foram rodadas regressões, agrupando todos os anos da amostra, e utilizados como variáveis independentes, além das já apresentadas, o salário mínimo real, a taxa de inflação (INPC), a produtividade do trabalho na agricultura, a taxa de desemprego e o câmbio real dos meses de referência das PNADs ${ }^{9}$.

\section{Resultados e discussões}

As estimativas das equações salariais estão na Tabela 4. Primeiramente, destaca-se que os coeficientes estimados são significativos a $1 \%$, com exceção da estimativa da razão de Mills invertida $(\lambda)$, nos anos de 1995 e 2005. Os coeficientes estimados para a variável educação indicam que o diferencial salarial, em relação às pessoas sem instrução ou com menos de um ano de estudo (Nível 1), reduziu no período analisado, especialmente na última década. Nota-se,

${ }^{9}$ O salário mínimo, a taxa de inflação (INPC), a taxa de câmbio, a taxa de variação anual do PIB brasileiro e o PIB do setor agrícola foram obtidos no site: http://www. ipea.gov.br. O salário mínimo e a taxa de câmbio média mensal foram deflacionados pelo INPC (set/2004=100). A produtividade do trabalho agrícola resultou da divisão do PIB do setor agrícola pelo pessoal ocupado neste setor, obtido nas PNADs. A taxa de desemprego também foi obtida das PNADs, da divisão da população economicamente desocupada pela população economicamente ativa. 
conforme o esperado, que quanto maior o nível educacional, maior é o salário esperado, ou seja, em 2005, os diferenciais salariais eram iguais a $6,46 \%, 13,99 \%, 24,94 \%, 46,57 \%$ e $581,13 \%$, para os Níveis $2,3,4,5$ e 6 , respectivamente, em relação ao nível $1^{10}$. Assim, verifica-se que ocorreu uma redução nos diferenciais salariais, considerando a variável educação, uma vez que, em 1981, esses diferenciais eram iguais a $19,38 \%, 48,29 \%, 93,35 \%, 275,8 \%$ e $737,48 \%$, respectivamente. Com isso, pode-se dizer que ocorreu uma redução dos diferenciais salariais entre os trabalhadores mais e menos qualificados no setor agrícola brasileiro, uma vez que as estimativas para a variável educação indicam que os salários pagos para os empregados com menos anos de estudos se aproximou daqueles pagos aos empregados com mais anos de estudos. Esse resultado é diferente do observado para a economia brasileira, conforme os trabalhos de Green et al. (2001) e Arbache et al. (2004), nos quais os prêmios salariais dos trabalhadores mais qualificados aumentaram após a abertura comercial.

A idade para o qual o salário esperado é máximo teve poucas oscilações ao longo do período analisado, ou seja, em 1981 era de 42,6 anos, em média, e em 2005, de 47,3 anos. No entanto, como o esperado, observa-se um pequeno aumento nos anos médios, acompanhando o envelhecimento da população brasileira no período.

Os diferenciais salariais de gênero, que no início do período estavam em $22,8 \%$, situam-se em um nível inferior até a segunda metade da década de 1990, mas voltam a aumentar, atingindo o valor de $27,5 \%$, em 2005. Dessa forma, na comparação entre 1981 e 2005, pode-se observar um aumento dos diferenciais salariais dos homens em relação às mulheres, na agricultura brasileira.

Os diferenciais salariais regionais têm uma tendência de crescimento no início do período analisado, mas pode-se notar uma redução na última década. Porém, essa redução não foi suficiente para superar o aumento anterior. Assim, em média, os diferenciais regionais aumentaram. Em 2005, os diferenciais salariais das regiões

${ }^{10}$ Por exemplo, se o coeficiente da variável binária associada à variável sexo masculino for $b$, então, a diferença percentual no salário esperado mediano do sexo masculino em relação ao feminino será igual a $100[\exp (b)-1] \%$. 
Norte, Sudeste (excluindo São Paulo), Sul e Centro-Oeste e do estado de São Paulo em relação à região Nordeste eram de 51,6\%, 20,1\%, $41,6 \%, 63,9 \%$ e 48,2\%, respectivamente. Ao final do período, a região Centro-Oeste está com o maior diferencial salarial em relação ao Nordeste ${ }^{11}$.

Tabela 4. Equações salariais estimadas para as pessoas empregadas na agricultura, $($ set $/ 2005=100), 1981-2005$

\begin{tabular}{lcccc}
\hline & 1981 & 1985 & 1995 & 2005 \\
\hline Constante & 3,403 & 3,496 & 3,663 & 3,844 \\
Escolaridade & & & & \\
$\quad$ Nível 2 & 0,177 & 0,091 & 0,161 & 0,063 \\
$\quad$ Nível 3 & 0,394 & 0,209 & 0,351 & 0,131 \\
$\quad$ Nível 4 & 0,659 & 0,470 & 0,600 & 0,223 \\
$\quad$ Nível 5 & 1,324 & 0,894 & 1,180 & 0,382 \\
$\quad$ Nível 6 & 2,125 & 2,266 & 2,361 & 1,919 \\
Idade/10 & 0,508 & 0,413 & 0,306 & 0,327 \\
(Idade/10) & $-0,059$ & $-0,044$ & $-0,033$ & $-0,035$ \\
Sexo masculino & 0,205 & 0,216 & 0,147 & 0,243 \\
Região & & & & \\
$\quad$ Norte & 0,482 & 0,600 & 0,523 & 0,416 \\
MG + ES + RJ & 0,094 & 0,179 & 0,248 & 0,183 \\
SP & 0,414 & 0,512 & 0,565 & 0,393 \\
Sul & 0,072 & 0,182 & 0,212 & 0,348 \\
$\quad$ Centro-Oeste & 0,243 & 0,393 & 0,381 & 0,494 \\
Tempo semanal de trabalho & & & & \\
$\quad 40$ a 44 horas & 0,296 & 0,332 & 0,359 & 0,469 \\
45 a 48 horas & 0,437 & 0,442 & 0,485 & 0,548 \\
$\geq 49$ horas & 0,505 & 0,533 & 0,556 & 0,637 \\
Carteira de trabalho assinada & 0,441 & 0,395 & 0,288 & 0,443 \\
Lâmbida & 0,330 & 0,159 & 0,411 & $-0,063$ \\
$R^{2}$ & 35,39 & 37,86 & 39,58 & 49,13 \\
$N \quad$ & 14.172 & 17.960 & 8.904 & 9.325 \\
\hline
\end{tabular}

Fonte: Elaborado com base nas PNADs. ${ }^{a}$ Os coeficientes estimados em itálico não foram estatísticamente significativos a um nível de $1 \%$.

${ }^{11}$ Deve-se ressaltar que a região Norte também apresenta um alto diferencial salarial em relação à região Nordeste. No entanto, os dados das PNADs excluem a área rural dessa região, o que deve estar superestimando o valor desses diferenciais, por incluir apenas as pessoas ocupadas no setor agrícola residentes nas áreas urbanas. 
Figura 2. Estimativas dos diferenciais salariais ${ }^{a}$ das pessoas ocupadas na agricultura, segundo os fatores educação (a), gênero (c), região (d), tempo semanal de trabalho (e) e carteira de trabalho; e a idade para o qual o salário estimado esperado é máximo (b), 1981-2005

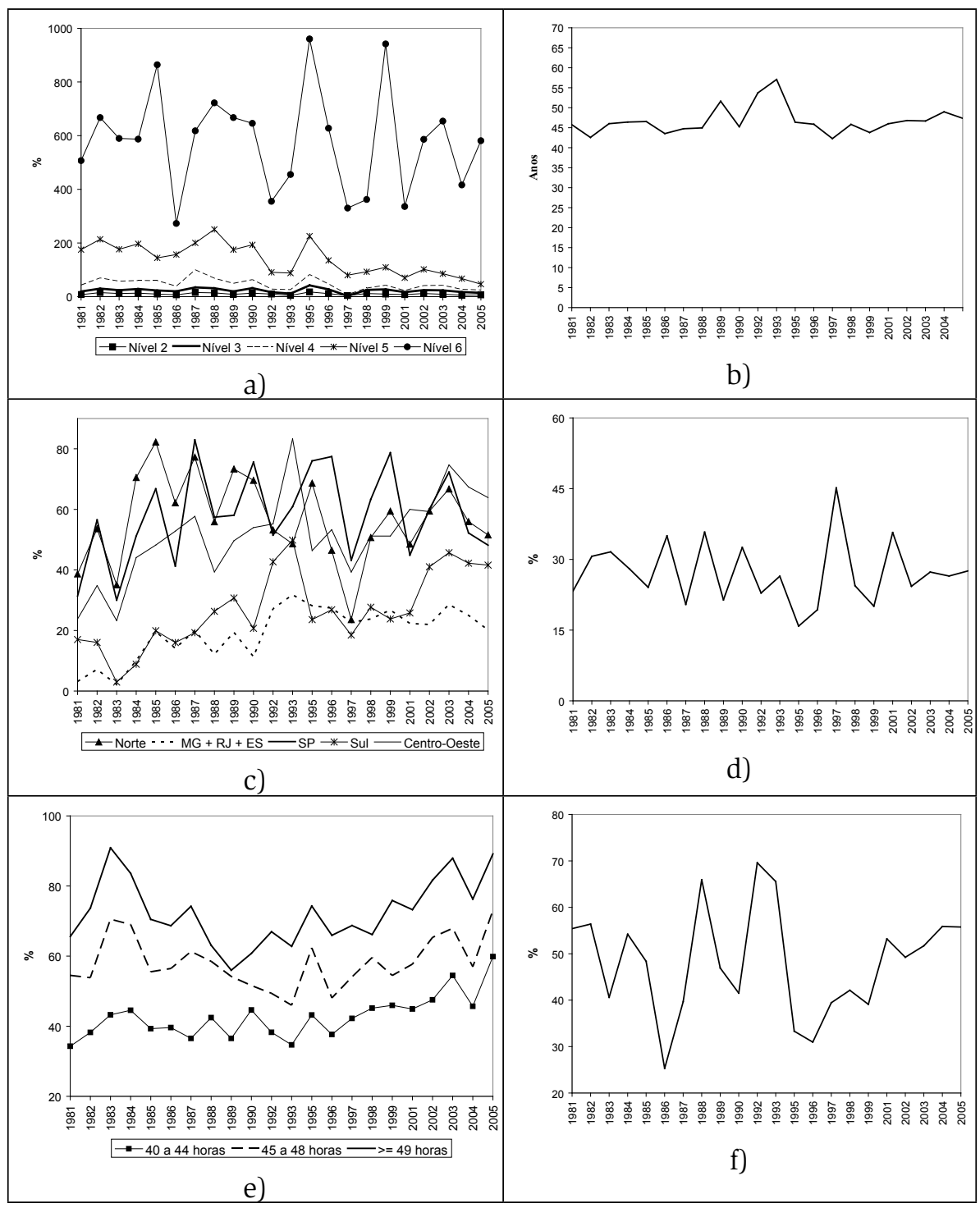

Fonte: Elaborado com base nas PNADs. ${ }^{\text {a }}$ Os valores estimados $(b)$ estão em percentuais, obtidos através de: $100[\exp (b)-1] \%$. 
Como o esperado, quanto maior o tempo semanal de trabalho, maior é o diferencial salarial em relação à categoria tomada como base (15 a 39 horas de trabalho semanal). Além disso, nota-se que os diferenciais salariais para esse fator apresentam uma nítida tendência de crescimento no período.

Os resultados sugerem que os diferenciais salariais dos empregados com carteira de trabalho caíram em 1995, com a estabilização da economia, mas voltaram a subir no final do período. Em 1981, os empregados na agricultura brasileira com carteira de trabalho tinham um salário mediano 55,5\% superior ao daqueles sem carteira e, em 2005, um salário mediano 55,9\% maior.

Após obter as equações salariais, pode-se calcular a decomposição do diferencial salarial entre os anos 1981 e 2005, na componente devido às mudanças nos coeficientes (diferencial não explicado) e na parcela originada da mudança das características ou da composição dos indivíduos (diferencial explicado), conforme a Tabela 5 .

A parte superior da Tabela mostra a contribuição individual de cada variável na decomposição salarial. Por exemplo, a variável educação (Nível 2) reduziu o diferencial salarial de salários entre 1981 e 2005, em que a menor parcela $(-0,004)$ deve-se à mudança na composição das pessoas ocupadas no setor agrícola, ou seja, à redução dos indivíduos com alguma educação elementar (Nível 2). Por outro lado, a maior parcela $(-0,036)$ corresponde à parcela não explicada, ou seja, a mudanças nos coeficientes entre os anos de 1981 e 2005.

As contribuições percentuais de cada grupo de variáveis podem ser observadas na parte inferior da Tabela 5. A proporção explicada pelo modelo corresponde a $84 \%$ do total, e as variáveis educação, idade, tempo semanal de trabalho e carteira de trabalho influenciaram positivamente para o diferencial de salários entre 1981 e 2005, enquanto as variáveis gênero e região, negativamente. Os outros 16\% corresponde à parte não explicada, ou seja, devem-se a diferenças na remuneração entre 1981 e 2005. Assim, se os indivíduos em 1981 fossem remunerados como os de 2005 , o diferencial de salário diminuiria $16 \%$, restando apenas o efeito composição. 
Tabela 5. Decomposição de Oaxaca - Heckman, 1981 e 2005

\begin{tabular}{|c|c|c|c|}
\hline \multicolumn{4}{|c|}{ Contribuição individual das variáveis } \\
\hline & \multicolumn{3}{|c|}{ Variação Percentual } \\
\hline & Explicada & Não Explicada & Total \\
\hline Constante & & 0,441 & 0,441 \\
\hline \multicolumn{4}{|l|}{ Escolaridade } \\
\hline Nível 2 & $-0,004$ & $-0,036$ & $-0,041$ \\
\hline Nível 3 & 0,021 & $-0,048$ & $-0,027$ \\
\hline Nível 4 & 0,017 & $-0,006$ & 0,011 \\
\hline Nível 5 & 0,016 & $-0,005$ & 0,011 \\
\hline Nível 6 & 0,003 & 0,000 & 0,003 \\
\hline Idade/10 & 0,048 & $-0,601$ & $-0,553$ \\
\hline$(\text { Idade } / 10)^{2}$ & 0,021 & 0,344 & 0,365 \\
\hline Sexo masculino & 0,007 & 0,033 & 0,040 \\
\hline \multicolumn{4}{|l|}{ Região } \\
\hline Norte & 0,005 & 0,000 & 0,004 \\
\hline $\mathrm{MG}+\mathrm{ES}+\mathrm{RJ}$ & $-0,006$ & 0,023 & 0,016 \\
\hline SP & $-0,004$ & $-0,003$ & $-0,007$ \\
\hline Sul & $-0,014$ & 0,041 & 0,026 \\
\hline Centro-Oeste & 0,016 & 0,020 & 0,036 \\
\hline \multicolumn{4}{|l|}{ Tempo semanal de trabalho } \\
\hline 40 a 44 horas & 0,072 & 0,035 & 0,107 \\
\hline 45 a 48 horas & $-0,066$ & 0,040 & $-0,026$ \\
\hline$\geq 49$ horas & $-0,041$ & 0,047 & 0,005 \\
\hline Carteira de trabalho assinada & 0,085 & 0,000 & 0,085 \\
\hline Lâmbida & 0,015 & $-0,286$ & $-0,272$ \\
\hline Total & 0,189 & 0,036 & 0,225 \\
\hline \multicolumn{4}{|c|}{ Contribuição por grupos de variáveis } \\
\hline & Explicada & Não Explicada & Total \\
\hline Constante & 0,0 & 196,4 & 196,4 \\
\hline Educação & 23,4 & $-42,7$ & $-19,2$ \\
\hline Idade & 30,6 & $-114,6$ & $-84,0$ \\
\hline Sexo & $-1,6$ & 35,3 & 33,7 \\
\hline Região & $-15,8$ & 54,1 & 38,3 \\
\hline Tempo semanal de trabalho & 37,8 & 0,1 & 38,0 \\
\hline Carteira de trabalho assinada & 6,5 & $-127,5$ & $-120,9$ \\
\hline Total & 84,0 & 16,0 & 100,0 \\
\hline
\end{tabular}

Fonte: Elaborado com base nas PNADs. 
No entanto, considerando a parcela explicada e a não explicada, as variáveis educação, idade e carteira de trabalho têm reduzido os diferencias salariais entre 1981 e 2005 e, por outro lado, as variáveis gênero, região geográfica e tempo semanal de trabalho, têm aumentado-os.

Por fim, nota-se que, a constante capta um grande aumento do diferencial através dos coeficientes, não explicado pelas variáveis utilizadas na regressão. Ressalta-se que a constante reflete 0 comportamento do grupo de referência, ou seja, empregadas analfabetas ou com menos de um ano de estudo no setor agrícola, residentes na região Nordeste, sem carteira de trabalho assinada e trabalhando de 15 a 39 horas semanais.

Os resultados das estimativas com as informações de todos os anos e a inclusão das variáveis macroeconômicas estão na Tabela 6. Primeiramente, destaca-se que os respectivos coeficientes estimados estão com os sinais de acordo com o esperado e são estatisticamente significativos, com exceção do coeficiente para a variável produtividade.

Os efeitos do salário mínimo sobre o mercado de trabalho podem ser sintetizados em efeito preço e efeito farol, conforme Foguel (1998). O primeiro está associado ao fato de esta representar um preço do fator trabalho não-qualificado, no mercado de trabalho formal. Já o fato de o salário mínimo servir como um “indexador” para vários preços na economia e no mercado de trabalho justifica o segundo efeito. Assim, não é possível estabelecer um sentido para o efeito do salário mínimo nos salários do setor agrícola. No entanto, é possível interpretar o sinal positivo estimado, uma vez que grande parcela dos salários no setor agrícola é diretamente determinada pelo salário mínimo nacional.

Conforme a teoria econômica, tanto a inflação quanto o desemprego têm um efeito negativo sobre os salários agrícolas. A inflação reduz os salários reais, uma vez que retira o poder de compra destes e a taxa de desemprego reduz o poder de barganha dos trabalhadores no mercado de trabalho. 
Tabela 6 - Equações salariais estimadas para as pessoas empregadas na agricultura (set/2005 = 100), 1981-2005

\begin{tabular}{|c|c|c|c|c|c|c|}
\hline & $(1)$ & $(2)$ & (3) & (4) & $(5)$ & (6) \\
\hline Constante & 4,198 & 2,209 & 2,559 & 2,561 & 3,618 & 3,629 \\
\hline \multicolumn{7}{|l|}{ Escolaridade } \\
\hline Nível 2 & 0,095 & 0,113 & 0,105 & 0,105 & 0,136 & 0,129 \\
\hline Nível 3 & 0,161 & 0,208 & 0,192 & 0,190 & 0,257 & 0,238 \\
\hline Nível 4 & 0,339 & 0,412 & 0,390 & 0,387 & 0,456 & 0,420 \\
\hline Nível 5 & 0,919 & 1,040 & 1,023 & 1,020 & 1,016 & 0,967 \\
\hline Nível 6 & 1,972 & 2,153 & 2,143 & 2,139 & 2,065 & 2,006 \\
\hline Idade/10 & 0,075 & 0,075 & 0,072 & 0,072 & 0,089 & 0,088 \\
\hline$(\text { Idade } / 10)^{2}$ & $-0,001$ & $-0,001$ & $-0,001$ & $-0,001$ & $-0,001$ & $-0,001$ \\
\hline Sexo masculino & 0,346 & 0,250 & 0,245 & 0,247 & 0,324 & 0,348 \\
\hline \multicolumn{7}{|l|}{ Região } \\
\hline Norte & 0,411 & 0,481 & 0,474 & 0,471 & 0,473 & 0,454 \\
\hline $\mathrm{MG}+\mathrm{ES}+\mathrm{RJ}$ & 0,241 & 0,291 & 0,294 & 0,293 & 0,251 & 0,243 \\
\hline SP & 0,563 & 0,634 & 0,641 & 0,639 & 0,570 & 0,557 \\
\hline Sul & 0,331 & 0,343 & 0,348 & 0,347 & 0,308 & 0,309 \\
\hline Centro-Oeste & 0,418 & 0,452 & 0,450 & 0,449 & 0,454 & 0,451 \\
\hline \multicolumn{7}{|l|}{$\begin{array}{l}\text { Tempo semanal de } \\
\text { trabalho }\end{array}$} \\
\hline 40 a 44 horas & 0,285 & 0,305 & 0,310 & 0,309 & 0,311 & 0,316 \\
\hline 45 a 48 horas & 0,473 & 0,481 & 0,486 & 0,486 & 0,421 & 0,420 \\
\hline$\geq 49$ horas & 0,479 & 0,496 & 0,502 & 0,503 & 0,446 & 0,446 \\
\hline $\begin{array}{l}\text { Carteira de trabalho as- } \\
\text { sinada }\end{array}$ & 0,307 & 0,332 & 0,327 & 0,328 & 0,362 & 0,358 \\
\hline Salário Mínimo & & 0,008 & 0,007 & 0,007 & 0,009 & 0,009 \\
\hline Inflação & & & $-0,009$ & $-0,009$ & $-0,023$ & $-0,024$ \\
\hline Produtividade & & & & 0,001 & $-0,073$ & $-0,116$ \\
\hline Desemprego & & & & & $-0,132$ & $-0,127$ \\
\hline Câmbio & & & & & & 0,073 \\
\hline Lambda & 0,202 & 0,372 & 0,375 & 0,367 & 0,261 & 0,225 \\
\hline$R^{2}$ & 0,1481 & 0,192 & 0,1981 & 0,1981 & 0,2455 & 0,2486 \\
\hline$N$ & 278.269 & 278.269 & 278.269 & 278.269 & 278.269 & 278.269 \\
\hline
\end{tabular}

Fonte: Elaborado com base nas PNADs. ${ }^{a}$ Os coeficientes estimados em itálico não foram estatísticamente significativos a um nível de $5 \%$.

Por outro lado, o sinal esperado para a produtividade do trabalho é positivo. Contudo, o sinal estimado foi positivo apenas no modelo (3), em que não foi estatisticamente significativo. Já nos dois últimos 
modelos, o sinal estimado foi negativo e estatisticamente significativo. Portanto, esse fato sugere que os aumentos de produtividade no setor agrícola não estão sendo traduzidos em aumentos dos salários reais do setor. Deve-se salientar também que a inclusão dessa variável não alterou o coeficiente de determinação, o que sugere que ela, em média, não tem efeitos significativos sobre os salários reais do setor $^{12}$.

Teoricamente, espera-se que uma desvalorização cambial tenha efeitos positivos para os salários, ou seja, uma desvalorização cambial aumenta a competitividade dos produtos nacionais, elevando a demanda interna por mão-de-obra, o que propicia um aumento dos salários médios, considerando uma oferta de mão-de-obra relativamente inelástica no curto prazo.

Com isso, é possível dizer que a inclusão das variáveis macroeconômicas foi significativa para a explicação do comportamento dos salários dos empregados do setor agrícola, além das variáveis do modelo básico (educação, experiência, sexo, região, tempo semanal de trabalho e carteira de trabalho), definido na equação (2), aumentando o coeficiente de determinação de $14,8 \%$ para $24,7 \%$.

\section{Considerações finais}

Este trabalho objetivou analisar a evolução dos diferenciais e dos determinantes salariais agrícolas. Seguindo a tendência observada na economia brasileira, a desigualdade salarial no setor agrícola vem reduzindo notadamente a partir do início da década de 1990. Em 2005, o índice de Gini para o setor foi de 0,353. Deve-se ressaltar que a desigualdade na agricultura é menor do que a observada no restante da economia ao longo de todo o período, em que, por exemplo, o índice de Gini foi de 0,486 no ano de 2005.

\footnotetext{
${ }^{12}$ Souza Netto \& Curado (2005) analisaram a relação entre produtividade do trabalho e os salários reais na indústria de transformação, na década de 1990. Apesar de estimarem uma relação positiva entre estas duas variáveis, por meio do modelo de regressão linear, as séries não apresentaram uma relação de equilíbrio de longo prazo, pela análise de co-integração, método mais adequado, pois as séries não eram estacionárias. Com isso, para os autores, os ganhos de produtividade podem ter sido apropriados apenas em parte pelos salários, outra pelos lucros ou reinvestidos no setor.
} 
Os resultados também sugerem que as variáveis educação, idade e carteira de trabalho reduziram os diferencias salariais entre 1981 e 2005 e, por outro lado, as variáveis gênero, região geográfica e tempo semanal de trabalho, para aumentá-los. Com isso, ressalta-se a redução dos diferenciais salariais entre os trabalhadores mais e menos qualificados no setor agrícola brasileiro.

A inclusão de variáveis macroeconômicas entre os determinantes salariais mostrou-se estatisticamente significativa na explicação dos diferenciais salariais. O salário mínimo e o comportamento do PIB tiveram reflexos positivos nos salários esperados, enquanto a produtividade do trabalho agrícola, a inflação, o desemprego e a taxa de câmbio tiveram reflexos negativos.

Por fim, este trabalho sugere estudos mais detalhados sobre o comportamento da produtividade no setor agrícola, como por exemplo, por cultura e/ou região geográfica, uma vez que as estimativas indicam que, para o conjunto do setor, os aumentos de produtividade não estão sendo repassados para os salários.

\section{Referências bibliográficas}

ATTANASIO, O.,GOLDBERG, P.K., PAVENIK, N. Trade reforms and wage inequality in Colombia. NBER, Working Paper, 2003, 51p.

ARBACHE, J. S; DICKERSON; GREEN, F. Trade liberalisation and wages in developing countries. The Economic Journal, v. 114, n.493, 2004, p. 73-96.

BACHA, C. J. C. Determinação dos salários na agricultura. Revista Brasileira de Economia, v. 45, n. 3, 1991, p. 441-471.

BALSADI, O. V. Qualidade do emprego na agricultura brasileira no período 2001-2004 e suas diferenciações por culturas. Revista de Economia e Sociologia Rural, v.45, n.2, 2007, p.409-444.

BEYER, H; ROJAS, P; VERGARA; Trade liberalization and wage inequality. Journal of Development Economics, v. 59, 1999, p.103-123.

BLINDER, A. S. Wage discrimination: reduced form and structural variables. Journal of Human Resources, n. 8, p. 436-455, 1973. 
CRAGG, M. I.; EPELBAUM, M. Why has wage dispersion grown in México? Is it the incidence of reforms or the growing demand for skills? Journal of Development Economics, v. 51, 1996, p. 99-116.

COELHO, A.M; CORSEUIL, C.H. Diferenciais salariais no Brasil: um breve panorama. IPEA, ago, 2002, p. 21. (texto para discussão n. 898)

FEENSTRA, R. C.; GORDON, H.H. Globalization, outsourcing and wage inequality. American Economic Review Papers and Proceedings, v. 86, n.2, 1996, p. 240-45.

FOGUEL, M. N. Uma avaliação dos efeitos do salário mínimo sobre o mercado de trabalho no Brasil. Rio de Janeiro. IPEA, 1998, 20p. (Texto para discussão, no. 561)

GINDLING, T.H.; ROBBINS, D. Patterns and sources of changing wage inequality in Chile and Costa Rica during structural adjustment. World Development, v.29, n.4, 2001, p. 725-745.

GREEN, F., DICKERSON, A., ARBACHE, J. S. A picture of wage inequality and the allocation of labor through a period of trade liberalization: the case of Brazil, World Development, v. 29, n. 11, 2001, p. 1923-1939.

GREENE, W. H. Econometric Analysis. New Jersey: Pearson Education, $5^{\text {a }}$ ed. 2002.

HANSON, G; HARRISON, A. Trade liberalization and wage inequality in México. Industrial and Labor Relations Review, v. 52, n.2, 1999, p. 271-288.

HOFFMANN, R. Distribuição de renda: medidas de desigualdade e pobreza. São Paulo: Editora da Universidade de São Paulo, 1998.

HECKMAN, J. Sample selection bias as a specification error. Econometrica, n. 47, jan., 1979, p. 679-694.

HELFAND, S. M, REZENDE, G.C. A agricultura brasileira nos anos 1990: o impacto das reformas de políticas. In: CONCEIÇÃO, J. C. \& GASQUES, J. G. (org). Transformações da Agricultura e Políticas Públicas. Brasília, 2001, p.247-301. 
ISTAKE, M.; BACHA, C. J. C.. Comportamento do Emprego na Agropecuária do Paraná. Revista de Economia e Sociologia Rural, v. 37, n. 4, p. 71-102, 1999.

MACHIN, S.; REENEN, J. V. Technology and changes in skill structure: evidence from seven OECD Countries. The Quarterly Journal of Economics, 1998, v. 113, p. 1215-1244.

MACHADO, A. F.; JAYME, F.G. Liberalização comercial: impactos sobre a composição do emprego no Brasil. In: WAJNMAN, S.; MACHADO, A.F. (org). Mercado de trabalho: uma análise a partir das pesquisas domiciliares no Brasil. Belo Horizonte: Editora UFMG, 2003, p.267-292.

MENEZES-FILHO, N.; FERNANDES, R.; PICCHETTI; P. A evolução da distribuição de salários no Brasil: fatos estilizados para as décadas de 80 e 90. In: HENRIQUES, R. (org.). Desigualdade e pobreza no Brasil. Rio de Janeiro: IPEA, 2000, p. 231-250.

OAXACA, R. Male-Female wage differentials in urban labour markets. International Economic Review - v. 14, no. 3, out. 1973, p. 693709 .

ROMAGUERA, P. Wage differentials and efficiency wage models: Evidence from the chilean economy. Working Paper, n. 193, 38p, 1991.

SACHS, J.; SHATZ, H. Globalization and the U.S. labor market: U.S. trade with developing countries and wage inequality. The American Economic Review, v.86, n. 2, 1996, p. 234-239.

STADUTO, J. A. R., BACHA, C. J. C.; BACCHI, M. R. P. Determinação dos salários na agropecuária brasileira. Pesquisa e planejamento econômico, v.32, n.2, 2002, p.285-321

SOARES, F. V. The impact of trade liberalisation on the informal sector in Brazil. Brasília: Departamento de Economia da Universidade de Brasília. 2004, 42p. (Seminário no.05/04)

SOUZA NETO, C. R.; CURADO, M. L. Produtividade do Trabalho, Salários Reais e Desemprego na Indústria de Transformação na Década de 90: Teoria e Evidência. Revista de economia contemporânea, Rio de Janeiro, v. 9, n. 3, p. 485-508, 2005. 
WOOD, A. Openness and wage inequality in developing countries: The Latin American challenge to East Asian Conventional wisdom. World Bank Economic Review, n. 11, 1997, p. 33-58 